\title{
VALIDACIÓN DE LA RESPUESTA DEL PASTO TRANSVALA (DIGITARIA ERIANTHA) EN PRODUCCIÓN Y CALIDAD DE HENO BAJO RIEGO
}

\author{
Jorge L. Morales González", Vidal Acuña Redondo ${ }^{1}$
}

\begin{abstract}
RESUMEN
El presente trabajo validó la tecnología para la producción de heno de pasto Transvala en sistemas bajo riego. Para esto se evaluó, a nivel de finca, el método de siembra del cultivo, el establecimiento hasta producción, y los procesos de manejo de lotes para henificación, costos y rentabilidad. Se validó también, la respuesta del cultivo a la fertilización nitrogenada y al manejo de 45 a 50 días de rebrote. Se evaluó, como valor agregado a la tecnología original, la comparación entre dos fuentes de nitrógeno y su dosificación, así como, el número de riegos, según las condiciones físicas del suelo. Se corroboraron los resultados de la estación experimental plenamente en lo que se refiere a lo mencionado en este párrafo.

Los resultados de esta validación indicaron que la tecnología desarrollada en la Estación Experimental Enrique Jiménez Núñez, se aplica comercialmente con ligeras modificaciones. El método de establecimiento se mantiene igual, más bien reduciendo un poco la intensidad de ciertas labores, a causa de la oportunidad de este cultivo para utilizar la infraestructura del arroz sin mayores problemas, con una reducción favorable en costos de establecimiento. Se reproduce la observación, de que con esta tecnología se logra un autocontrol de malezas, al no tolerar éstas el corte frecuente del cultivo.
\end{abstract}

El proceso de validación permitió determinar que los niveles de fertilizante no deben sobrepasar los $200 \mathrm{~kg}$ de N/ha/corte ya que se evidencia la posibilidad de incrementar la concentración de sustancias contaminantes en el suelo, tales como los nitratos. Este resultado es consecuente con los niveles de respuesta biológica y económica indicados por la tecnología, donde previo al presente estudio se recomendaba entre 65 y $100 \mathrm{~kg}$ de N/ ha/ corte.

Se logró comprobar los rendimientos, calidad del producto, costos y rentabilidad de la actividad sobre el $80 \%$, lo que permitió concluir la factibilidad biológica, económica y ambiental para proceder a transferir esta tecnología.

Palabras clave: Riego, fertilizante nitrogenado, nitratos, costos, rendimiento, rentabilidad

1 Instituto Nacional de Innovación y Transferencia en Tecnología Agropecuaria (INTA), Costa Rica. Correo electrónico: jmorales@inta.go.cr 


\section{INTRODUCCIÓN}

Investigaciones realizadas en la estación experimental Enrique Jiménez Núñez (Morales et al. 2006 a y b) han demostrado el potencial del riego para la producción de heno de pasto Transvala de calidad superior al heno comercial, producido en condiciones de secano. Las características ideales que posee esta especie forrajera para la henificación, se ven potenciadas con el riego, la fertilización nitrogenada y el manejo del rebrote de 45 a 50 días.

La tecnología desarrollada indicó, que es posible obtener cuatro cortes de este pasto, para henificación. El primero, similar al de los sistemas de producción de secano, se obtiene a inicios del verano, entre la tercera semana de noviembre y la primera de diciembre, dependiendo de la fecha en que este cambio de estación climática ocurra.

En este primer corte se obtienen entre 300 a 700 pacas, dependiendo si durante el veranillo de San Juan y la canícula anterior se pudo henificar y si en el invierno se sacó o no, forraje para la venta de semilla.

En todo caso, 500 pacas es el promedio estimado entre ambos extremos mencionados, que se obtienen debido a la acumulación de forraje durante tres a siete meses en el invierno, según los factores mencionados anteriormente, además, de que se aplique o no, fertilizante nitrogenado, práctica ocasional, realizada por algunos productores.

Como tal, esta es una producción alta de forraje, pero que bajo las condiciones de secano en que se desarrolla, es de una calidad muy baja, que en términos del contenido de proteína cruda, en general, anda por debajo de $5,8 \%$ y en algunas ocasiones, similar a la de paja de arroz de un 4,7\% (Morales 2003).

Los otros tres cortes son estrictamente, bajo el sistema de riego por gravedad existente y con el cual se han realizado los estudios base de la tecnología desarrollada, a un máximo de 50 días de rebrote, con una aplicación bianual de cuatro sacos por hectárea, de una fórmula completa como 10-30-10, lo cual permite suministrar al suelo aproximadamente $50 \mathrm{~kg}$ de $\mathrm{P} 2 \mathrm{O} 5$, para mantener la disponibilidad del fósforo en el suelo, ya que este nutriente es uno de los más limitantes en la zona (Gómez 2002).

La fertilización nitrogenada requerida, bajo las características de suelo de la estación experimental, tipo Mollisol (uno de los mejores, pero poco frecuente en el país), para lograr una producción rentable, de unas 300 pacas de $17 \mathrm{~kg}$ de peso cada una, es de 65 a $100 \mathrm{~kg}$ de nitrógeno por hectárea, dividida en tres aplicaciones iguales (Morales 2003). Se requieren además, tres riegos con lámina de agua de $10 \mathrm{~cm}$, cada 15 días, que van del día cero (inmediatamente después de la henificación y levantado de las pacas), el día 15 y día 30 aproximadamente, para dejar de 10 a 15 días para la utilización satisfactoria del fertilizante y el oreo del forraje, antes del siguiente corte. Las aplicaciones del fertilizante se hacen después del riego, en el proceso de drenaje, cuando la lámina de agua se encuentra a media vida y antes de desaparecer.

Con esta tecnología, desarrollada en la estación experimental, es posible producir, en cuatro cortes (primera semana de diciembre, finales de enero, mediados de marzo y finales de abril-principio de mayo) al menos 1400 pacas por hectárea, 500 de secano y 900 (300/ corte) de verano. No se considera el corte del veranillo de San Juan por lo errático, climatológicamente hablando, de este periodo. Esto significa que la producción de heno bajo riego triplica la cantidad y la calidad de la producción de secano. La calidad de este forraje en términos de proteína cruda es de aproximadamente $9 \%$ (Morales et al. 2006 b).

Los mismos estudios mencionados indicaron, un costo de establecimiento de $\varnothing 155400,00$ por hectárea (incluyendo costos de diseño y construcción de curvas de nivel, costo que no es necesario hacerlo en áreas donde ya existe la infraestructura como en el DRAT). El costo 
operativo total, en el ciclo de cuatro cortes, fue de $\phi 411500,00$. El ingreso por venta de 1400 pacas, a precios del año 2000 ( $\$$ 600/ paca), en una actividad comercial tradicional que no considera la calidad del forraje, es de $\varnothing 840000,00$, para un ingreso neto de $\varnothing 428$ 500,00 por hectárea, por ciclo de verano. Más adelante, en el presente artículo se mencionan los valores actualizados al 2011 de costos y rentabilidad de la tecnología.

Considerando que las condiciones de producción de los estudios realizados y las condiciones de los productores del DRAT, para quienes van dirigidos los resultados obtenidos, pueden diferir, se debe realizar la validación de los resultados en fincas de productores, paso necesario del desarrollo tecnológico y que determinará la factibilidad comercial, para la transferencia de la tecnología.

Por lo anterior, el objetivo del presente estudio fue: validar la viabilidad biológica, económica y ambiental, de producir heno de alta calidad, de pasto Transvala, en el Distrito de Riego Arenal-Tempisque.

\section{MATERIALES Y MÉTODOS}

\section{Ubicación del Estudio.}

El presente estudio se llevó a cabo en El Asentamiento Campesino La Falconiana, perteneciente al cantón de Bagaces, Guanacaste y ubicado a $15 \mathrm{~km}$ de la ciudad con el mismo nombre, el cual forma parte del Distrito de Riego Arenal Tempisque (DRAT). El periodo de ejecución fue de julio del 2000 a octubre del 2001.

Específicamente el estudio se ejecutó en las parcelas $\mathrm{N}^{\circ} 2$ y 13, de Floriberto Esquivel (q.d.D.g.) y Olider Rojas, respectivamente. Las características de los suelos en ambas parcelas se muestran en el Cuadro 1.
Cuadro 1. Características de los suelos de las parcelas donde se desarrolló el estudio de validación. Guanacaste, Costa Rica. 2000.

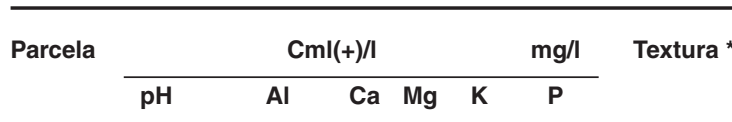

$\begin{array}{llllllll}\mathrm{N}^{\circ} 2 & 7,5 & 0,10 & 6,5 & 1,5 & 0,70 & 2 & \text { Franco-A }\end{array}$ 32-34-34

$N^{\circ} 13 \quad 7,1 \quad 0,10 \quad 7,6 \quad 2,0 \quad 0,8919$ Franco-A

- Franco-A= franco arcilloso. 28-36-36

La Figura 1 muestra el comportamiento del clima en la zona (Hancock y Hargreaves 1977), la cual se basó en un periodo de 6 años y en probabilidades de un $75 \%$ de que este patrón de lluvias pueda ocurrir.

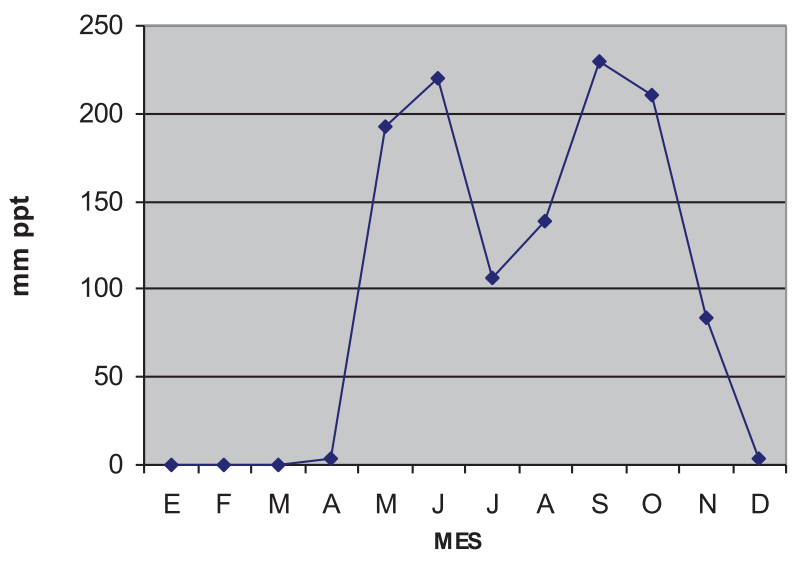

Figura 1. Patrón de lluvias con $75 \%$ probabilidades para el área de la E.E.E.J.N. Guanacaste, Costa Rica. 2000.

La temperatura promedio de la zona ha sido de $24,6^{\circ} \mathrm{C}$, la humedad relativa $91 \%$, las precipitaciones: máxima $2818 \mathrm{~mm}$, y, mínima $1018 \mathrm{~mm}$.

\section{Validación de los métodos y costos de establecimiento del pasto Transvala.}

\section{Establecimiento de las áreas del estudio.}

Se procedió a establecer 5 ha en la finca $\mathrm{N}^{\circ} 2$ y 1 ha en la finca $N^{\circ} 13$ respectivamente. Se 
aplicó el método de siembra y establecimiento que se utilizó en la estación experimental (Morales et al. 2003), para validar tanto el método, como los costos del mismo. Estas áreas sirvieron también, para las demás actividades de validación que se plantearon en el presente estudio.

\section{Validación de los niveles de producción, calidad y costos de producción de heno de pasto Transvala bajo riego.}

\section{Manejo de los lotes para henificación.}

El manejo de los lotes para henificación se siguió estrictamente según la tecnología que se desarrolló en estación experimental, aplicación de los riegos inmediatamente después de la henificación, fertilización de $100 \mathrm{~kg}$ de N/ ha en tres dosis iguales y edad de rebrote máxima de cincuenta días. Debido a que los suelos de las parcelas eran más livianos que los de la estación experimental, fue necesario variar el régimen a riegos semanales para mantener la humedad requerida. El último riego se hizo 10 días antes de la corta para el oreo suficiente del suelo. En total se realizaron cinco cortes, de noviembre del 2000 a agosto del 2001, incluidos el corte de secano del primer año y el del veranillo en julio del 2001.

\section{Análisis de la calidad del heno producido.}

Los diferentes lotes de heno que se produjeron fueron muestreados y enviados al laboratorio para la determinación de proteína cruda y fibra.

\section{Registro de costos e ingresos.}

Se llevó registro de actividades, insumos y costos, tanto del establecimiento de las áreas, como del manejo de los lotes para henificación y los procesos propiamente de henificación, que incluyeron servicio de maquinaria, mano de obra para levantar pacas y almacenarlas, número de pacas y precios de venta.
Validación de la respuesta del pasto a la fertilización nitrogenada, a 45 días de rebrote.

\section{Establecimiento del experimento.}

Para realizar este estudio, se estableció un experimento en parcelas pequeñas de $6 \mathrm{~m}^{2}$ (3 $\mathrm{m} \times 2 \mathrm{~m}$ ), sobre las áreas grandes establecidas para validar los resultados a nivel comercial. La parcela pequeña fue utilizada como unidad experimental, las cuales fueron marcadas el 14 de marzo del 2001. Para ello, se ubicaron dos bloques en la finca $\mathrm{N}^{\circ} 2$ y un bloque en la finca $N^{\circ} 13$, estas fincas se encuentran, aproximadamente, a $1 \mathrm{~km}$ de distancia entre una y otra. Aunque ambas fincas cosecharon su tercer corte para heno el 6-03-01, no fue sino hasta el 14 de marzo que se hizo el primer riego, por lo que ésta última es la fecha utilizada para establecer la edad de rebrote, que fue de 43 días, al 26 de abril.

\section{Diseño Experimental.}

Para este estudio se utilizó un diseño experimental de bloques con parcelas subdivididas, en un arreglo factorial con tres repeticiones (bloques), dos tipos de fuente de nitrógeno, urea y nitrato de amonio, como parcela principal, las cuales se fueron aplicadas en tres dosis de dos tipos, una dosis en tres aplicaciones de iguales cantidades y la otra en tres aplicaciones incrementales (1/ 6, 2/ 6 y 3/ 6), como segunda subparcela, además, se utilizaron seis niveles de fertilización 0, 100, 200, 300, 400 y 500 kg de nitrógeno por hectárea, como tercera subparcela. No se aplicó fórmula completa porque ésta se hizo en octubre anterior, en la etapa de establecimiento de las áreas grandes.

Para el análisis de los datos se utilizó el paquete estadístico SAS en un diseño en parcelas divididas. El análisis de varianza fue el siguiente: 
Cuadro 1. ANDEVA. Guanacaste, Costa Rica. 2000.

\begin{tabular}{cc}
\hline FUENTE DE & $\begin{array}{c}\text { GRADOS DE } \\
\text { LIBERTAD }\end{array}$ \\
VARIACION & $3-1=2$ \\
BLOQUE (B) & $2-1=1$ \\
FUENTE N (FN) & 2 \\
FNXB error a & $2-1=1$ \\
DOSIS (D) & 1 \\
DXFN & 4 \\
DXFNXBXFNXB error b & $6-1=5$ \\
NIVELES (N) & 5 \\
NXFN & 5 \\
NXD & 5 \\
NXFNXD & 40 \\
ERROR & $72-1=71$ \\
\hline TOTAL &
\end{tabular}

Este estudio de validación se aprovechó también, para investigar la respuesta del pasto, a fuentes diferentes de nitrógeno, lo cual no se había evaluado en los estudios anteriores. Considerando que, conforme la planta crece y tiene más biomasa, aumenta la necesidad y también la capacidad para aprovechar el fertilizante nitrogenado, se comparó la práctica, de dosificaciones en cantidades iguales, usual en cultivos, versus dosificaciones incrementales, esto con el fin de observar la posibilidad de mejorar la eficiencia en el uso del fertilizante nitrogenado, que es de alrededor de un $50 \%$, normalmente (Tisdale et al. 1985).

\section{Variables dependientes.}

Las variables dependientes fueron cuatro: producción de materia seca, porcentaje de materia seca, proteína cruda y fibra en el forraje.

\section{Muestreos.}

Se hizo un solo muestreo a los 43 días de rebrote (26-4-01). Para esto se cortó cada parcela a nivel del suelo. Se pesó todo la biomasa verde de la parcela con romana de reloj de $50 \mathrm{~kg}$. Posteriormente, se tomó una sub-muestra de aproximadamente $500 \mathrm{~g}$ de material fresco en bolsas de papel debidamente identificadas. Estas se llevaron a la oficina, donde fueron debidamente pesadas en romana granataria y se almacenaron en hieleras con hielo mientras se llevaron a laboratorio al cabo de dos días, en donde se sometieron a secado en horno a $60^{\circ} \mathrm{C}$ durante 72 horas y posteriormente se molieron en molino de martillo utilizando malla de $2 \mathrm{~mm}$.

\section{Análisis de Laboratorio.}

En el laboratorio de Piensos y Forrajes del INTA, las muestras se secaron en horno a $105^{\circ} \mathrm{C}$ durante 24 horas, para determinar materia seca. Las muestras se analizaron para contenido de proteína (\% PC), mediante el método de micro Kjeldahl, \% de fibra ácido detergente (FAD), (AOAC 1980) y fibra neutro detergente (FND) utilizando la metodología de Goering y Van Soest (1970).

\section{Distribución al azar de las edades de rebrote y niveles de fertilización, en los tres bloques.}

Los bloques ó repeticiones se ubicaron en sitios diferentes pero representativos de los campos de pasto Transvala disponibles. En cada bloque se asignaron las fuentes de nitrógeno al azar, luego se seleccionó al azar también, cada fuente de nitrógeno la cuál se aplicaría en dosis crecientes o dosis iguales, dentro de éstas últimas se asignaron al azar los niveles de nitrógeno a aplicar.

Las parcelas se separaron a lo largo y ancho entre sí, con callejones de 2 metros de ancho. La distribución de las parcelas con los tratamientos asignados en cada bloque se muestra en el siguiente cuadro (Cuadro 2). 
Cuadro 2. Distribución de los tratamientos. Guanacaste, Costa Rica. 2000.

\begin{tabular}{cccc|cccc|cccc}
\hline \multicolumn{3}{c|}{ BLOQUE 1 } & \multicolumn{3}{c|}{ BLOQUE 2 } & \multicolumn{4}{c}{ BLOQUE 3 } \\
\hline NA & U & NA & U & NA & U & NA & U & U & U & N & N \\
\hline DIN & DIN & DIG & DIG & DIG & DIG & DIN & DIN & DIN & DIG & DIG & DIN \\
6 & 3 & 2 & 4 & 1 & 3 & 6 & 1 & 2 & 4 & 5 & 6 \\
4 & 2 & 6 & 3 & 4 & 2 & 3 & 3 & 5 & 5 & 2 & 2 \\
5 & 4 & 3 & 6 & 6 & 4 & 5 & 2 & 1 & 6 & 6 & 5 \\
3 & 5 & 4 & 1 & 2 & 5 & 4 & 4 & 4 & 3 & 4 & 3 \\
1 & 2 & 1 & 5 & 3 & 6 & 1 & 5 & 3 & 2 & 3 & 1 \\
2 & 6 & 5 & 2 & 5 & 1 & 2 & 6 & 6 & 1 & 1 & 4 \\
\hline
\end{tabular}

$\mathrm{NA}=$ nitrato de amonio, $\mathrm{U}=$ urea, $\mathrm{DIN}=$ dosis incrementales, DIG = dosis iguales, $1=0$ nitrógeno, 2 $=100 \mathrm{~kg} \mathrm{~N} / \mathrm{ha}, 3=200 \mathrm{~kg} \mathrm{~N} / \mathrm{ha}, 4=300 \mathrm{~kg} \mathrm{~N} / \mathrm{ha}, 5=$ $400 \mathrm{~kg} \mathrm{~N} / \mathrm{ha}, 6=500 \mathrm{~kg} \mathrm{~N} / \mathrm{ha}$. Las parcelas en cada bloque mantuvieron el orden descrito en el cuadro, pero separadas entre sí por callejones de $2 \mathrm{~m}$.

Los niveles de fertilizante aplicados a cada parcela, correspondientes a los niveles por ha planeados, se dividieron en tres aplicaciones, la primera el día 20 de marzo, después del segundo riego, la segunda el 4 de abril y la última el 20 de abril, es decir, a los 6, 21 y 37 días de rebrote, según se describe en el siguiente cuadro (Cuadro 3).

Cuadro 3. Dosis de fertilizante aplicadas y $\mathrm{g}$ por parcela de $6 \mathrm{~m}^{2}$. Guanacaste, Costa Rica. 2000.

\begin{tabular}{ccccc}
\hline $\begin{array}{c}\text { Nitrato/ha } \\
\text { en kg. }\end{array}$ & $\begin{array}{c}\text { Nitrato de } \\
\text { amonio/ } \\
\text { parcela dosis } \\
\text { iguales en g. }\end{array}$ & $\begin{array}{c}\text { Nitrato de } \\
\text { amonio/ } \\
\text { parcela dosis } \\
\text { incrementales }\end{array}$ & $\begin{array}{c}\text { Urea/Parcela } \\
\text { dosis iguales } \\
\text { en g. }\end{array}$ & $\begin{array}{c}\text { Urea/Parcela } \\
\text { dosis } \\
\text { incrementales } \\
\text { en g. }\end{array}$ \\
\hline 0 & 0 & 0 & 0 & 0 \\
100 & 60 & $30 / 60 / 90$ & 44 & $22 / 44 / 65$ \\
200 & 119 & $60 / 119 / 179$ & 87 & $44 / 87 / 130$ \\
300 & 179 & $90 / 179 / 269$ & 130 & $65 / 130 / 196$ \\
400 & 239 & $120 / 239 / 358$ & 174 & $87 / 174 / 261$ \\
500 & 299 & $150 / 299 / 448$ & 217 & $109 / 217 / 326$ \\
\hline
\end{tabular}

Análisis de los niveles de nitritos y nitratos en las aguas de percolación, como consecuencia de la aplicación de fertilizantes nitrogenados en la producción de heno bajo condiciones de riego.

Los niveles de nitritos y nitratos en el suelo, son indicadoras de contaminación por causa, principalmente, de excesos en la fertilización nitrogenada. Para medir su presencia en las aguas de percolación, se diseñaron unos dispositivos especiales para recoger las aguas a $50 \mathrm{~cm}$ de profundidad en el suelo. Estos dispositivos y su colocación en el suelo se presentan en la Figura 2.

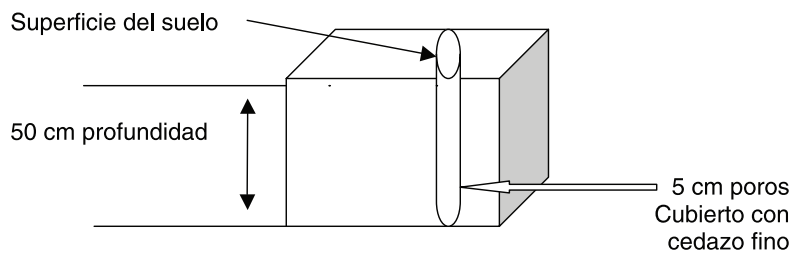

Figura 2. Diseño y colocación en el suelo del dispositivo recolector de agua para determinación de contaminantes. Guanacaste, Costa Rica. 2000.

Los dispositivos se diseñaron con tubos de pvc de 3/4, de $60 \mathrm{~cm}$ de largo con un tapón de hule en el fondo y una tapa plástica desmontable arriba. Debajo de la parte porosa quedaron $10 \mathrm{~cm}$ de tubo para el almacenaje del agua recolectada. El dispositivo fue colocado en un hueco delgado hecho a lo largo de los $50 \mathrm{~cm}$ de profundidad, con un barreno fabricado por el proyecto, con un tubo y manilla de hierro y punta de un taladro grueso para madera. Los tubos permanecieron en el suelo todo el tiempo de muestreo. El muestreo se realizó con una tripa corriente de plástico y succionando con una bomba de hule para mucosidades de bebe. Las muestras se vertieron en botellitas plásticas color ambar de $10 \mathrm{cc}$, debidamente rotuladas y mantenidas en refrigeración hasta su envío al laboratorio para su análisis.

Sobre las parcelas de validación que recibieron los tratamientos de dosis incrementales de nitrato de amonio, se estableció, el 27 de marzo del 2001, la evaluación para medir la 
presencia de nitritos y nitratos en las aguas de percolación. En cada una de ellas se colocaron los dispositivos para muestrear las aguas de percolación y determinar el contenido de nitritos y nitratos y así valorar el impacto ambiental de la nueva tecnología.

Dependiendo del momento de aplicación de los fertilizantes y de los muestreos de las aguas, es posible encontrar diferencias en el contenido de nitritos, inmediatamente después de la aplicación de fertilizantes nitrogenados los niveles de nitritos pueden ser muy superiores a los niveles críticos. Por ello, para subsanar la posibilidad de error, se hicieron dos muestreos, a los 15 días (4 y 18 de abril) después de las aplicaciones de fertilizantes y uno último cuatro días después de la aplicación de la última dosis de fertilizante, que fue la mayor en el tratamiento con dosis incrementales. De esta manera, se podría tener una mayor posibilidad de observar y detectar mejor, contaminaciones con mayor permanencia.

\section{RESULTADOS}

\section{Validación de los métodos y costos de establecimiento del pasto Transvala.}

En las parcelas de Olider Rojas y de Floriberto Esquivel se siguieron los métodos probados en la estación experimental Enrique Jiménez Núñez para el establecimiento del pasto Transvala. Se sembraron un total de 6 hectáreas.

Preparación del suelo. El suelo se preparó de forma convencional durante el veranillocanícula. El pase inicial de arado no fue necesario, pues estas parcelas se han sembrado continuamente de arroz, además, el tipo de suelos franco arcillosos, no requieren tal tratamiento. En el caso de la estación experimental si se requirió, porque se trataba de un área que tenía varios años sin cultivarse, era pastoreada y tenía una vegetación densa que a pesar de que se quemó con herbicidas, mantenía residuos radiculares densos y tallos, a nivel del subsuelo y suelo, respectivamente.
La infraestructura existente para el riego del cultivo del arroz, dividida en bancales con pendiente cero, bordos, canales regadores y de drenaje, se mantuvieron tales coma estaban, ya que para la henificación con este tipo de pasto, se adecuan perfectamente. Se dieron los dos pases de rastra. La nivelación consistió solamente en un pase de rufa, ya que ésta no se requiere tan fina como en el cultivo del arroz.

Siembra. La siembra se hizo con semilla de pasto Transvala, procedente de los campos de la Estación Experimental E.E.J.N., con una edad de 90 días de rebrote, lo cual garantizó la pega del material. Se utilizaron, aproximadamente, 3,5 t de semilla por hectárea. La semilla se hizo regada en el campo, luego se dio una pasada de rastra para incorporarla. Para este momento ya habían retornado las lluvias de una forma ligera lo que permitió perfectamente el manejo de la siembra aún con maquinaria de forma convencional, por lo que no fue necesario el riego para el establecimiento.

Manejo del establecimiento: fertilización y control de malezas.- A los 22 días de la siembra, se hizo una aplicación de fertilizante fórmula completa 12-24-12, a una tasa de tres sacos por ha, para ayudar al enraizamiento y establecimiento de los lotes. A los 60 días se hizo una aplicación de Basagran M60, para el control de coyolillo y otras malezas de hoja ancha y posteriormente se utilizó el control manual de las mismas. Al momento de la primera cosecha el 23 de noviembre (ese año el verano entró el 10 de noviembre), el contenido de malezas era relativamente alto, aproximadamente un $50 \%$, principalmente arroz voluntario (Oriza sativa), echinocloa (Echinocloa colonum) y la cyperacea pelo chino (Fimbristylis annua). Aquí se demostró lo observado en la estación experimental, que aunque se debe controlar malezas, aún cuando haya una proporción importante de ellas, por lo menos herbáceas como las mencionadas, conforme los cortes de henificación en el verano van sucediendo, éstas van desapareciendo, porque no soportan 
el régimen de corte del pasto Transvala. El método de establecimiento fue validado con éxito, ya que estos lotes estuvieron listos para entrar en producción a los 113 días de sembrados, en noviembre.

\section{Costos de establecimiento.}

El costo del establecimiento de pasto Transvala en finca resultó menor al de la estación experimental. La razón principal fue que en finca ya existía toda una infraestructura para riego, la cual no requirió ninguna modificación para la producción de heno con pasto Transvala. También, no fue necesario dar el pase de arado por las condiciones de un suelo trabajado en el cultivo de arroz continuamente. De aquí que los costos en finca se distribuyeron de la siguiente manera, según se muestra en el siguiente cuadro (Cuadro 4).

Cuadro 4. Costos por hectárea de siembra y establecimiento de pasto Transvala para la producción comercial de heno bajo riego en finca, comparado con Estación Experimental. Guanacaste, Costa Rica. 2000.

\begin{tabular}{|c|c|c|c|c|}
\hline Actividad & Unidades & Costo/ unidad $c$ & $\begin{array}{l}\text { Costo Total } \\
c \text { en finca }\end{array}$ & $\begin{array}{l}\text { Costo Total } \\
\text { c en Estación } \\
\text { Experimental }\end{array}$ \\
\hline $\begin{array}{l}\text { TOPOGRAFIA Y } \\
\text { CONSTRUCCION } \\
\text { DE BORDOS }\end{array}$ & & 20000 & & 20000 \\
\hline PASES DE ARADO & & 5000 & & $2=10,000$ \\
\hline PASE DE RASTRA & 3 & 2500 & 7500 & $4=10000$ \\
\hline PASES DE RUFA & 1 & 7000 & 7000 & 7000 \\
\hline $\begin{array}{c}\text { SEMILLA } \\
\text { (más transporte) }\end{array}$ & $3,5 \mathrm{t}$ & 19425 & 68000 & 68000 \\
\hline $\begin{array}{l}\text { MANO OBRA } \\
\text { SIEMBRA }\end{array}$ & 2 JORNALES & 3880 & 7760 & 7760 \\
\hline $\begin{array}{l}\text { MANO OBRA } \\
\text { APLICACIONES }\end{array}$ & 3 JORNALES & 3880 & 11640 & 11640 \\
\hline FERTILIZANTES & 3 qq 12-24-12 & 4200 & 12600 & 12600 \\
\hline HIERBICIDAS & 1 L Basagrán M60 & 6000 & 6000 & 6000 \\
\hline TOTAL & & & c120 500,00 * & $\varnothing 153000$ \\
\hline
\end{tabular}

*Los costos por hectárea, actualizados al 2011, en finca, son de $\varnothing 592$ 220,00 (Cuadro 1 en el anexo), aumentando el costo casi en un 500 \% en diez años. (Costos operativos al 2010 pueden observarse en cuadro 2 en el anexo). 
Validación de los niveles de producción, calidad y costos de producción de heno de pasto Transvala bajo riego.

La validación del comportamiento comercial de la tecnología de producción de heno de pasto Transvala se realizó sólo en la finca $\mathrm{N}^{\circ}$ 2, ya que por el pequeño tamaño del área de Transvala de la otra finca, se dificultó encontrar servicio de maquinaria de henificación que lo hiciera con la puntualidad que se requería.

\section{Producción}

En el siguiente Cuadro 5 se muestran las fechas y número de cortes, niveles de fertilización utilizados y el resultado económico logrado en la finca.

Cuadro 5. Manejo y producción alcanzado por Floriberto Esquivel durante el primer ciclo de producción. Guanacaste, Costa Rica. 2000.

\begin{tabular}{cccc}
\hline \multicolumn{2}{c}{$\begin{array}{c}\text { Fecha Siembra }= \\
\text { 2 agosto 2000 }\end{array}$} & \multicolumn{2}{c}{$\begin{array}{c}\text { Periodo Producción } \\
=9 \text { meses }\end{array}$} \\
\hline $\begin{array}{c}\text { FECHA } \\
\text { COSECHAS }\end{array}$ & $\begin{array}{c}\text { PRODUCCION } \\
\text { No PACAS }\end{array}$ & $\mathrm{kg} \mathrm{N} /$ ha & $\mathbf{N}^{\circ}$ PACAS/ ha \\
\hline NOV. 23 & 800 & & 160 \\
ENE. 3 & 930 & 99 & 186 \\
FEB. 20 & 1070 & 99 & 214 \\
ABR. 10 & 1300 & 99 & 260 \\
MAY. 27 & 1485 & 60 & 297 \\
AGO 18 & 975 & 58 & 195 \\
\hline TOTAL & $\mathbf{6 5 6 0}$ & $\mathbf{4 1 5}$ & $\mathbf{1 3 1 2}$ \\
\hline VALOR 4 600 & $\mathbf{3 9 3 6 0 0 0 ^ { * }}$ & & $\mathbf{7 8 7 ~ 2 0 0}$ \\
/ PACA & & & \\
\hline
\end{tabular}

* A valores actualizados al 2011 el valor de esta producción ( $\$ 1750 /$ paca) es de $\varnothing 11515000$ total * y de c2 296 000/ ha**

Se logró hacer los cortes cada 45-50 días y se aplicaron los niveles de fertilización recomendados. Fue necesario, sin embargo, un riego semanal y en algún momento hasta dos riegos por semana, como se previó, dadas las características edafológicas más francas de la zona que los suelos de la estación experimental. Como se puede observar en el Cuadro anterior, por obvias razones, las primeras cosechas después de la siembra del Transvala fueron de producción baja (y de baja calidad por el contenido alto de malezas). Conforme los cortes se fueron realizando, el cultivo se fue volviendo más denso y productivo. Para el caso en estudio, hasta en el quinto corte, se logró la meta de las 300 pacas/ ha. Estas pacas en promedio tuvieron un peso de $17 \mathrm{~kg}$, tamaño deseable para su comercialización (15 a 20 kg).

Para que las comparaciones resulten adecuadas, deben hacerse a partir del momento que se logre estabilizar la producción en 300 pacas/ ha. Debe considerarse también que las estimaciones de comparación se hacen sobre la base de cuatro cortes, sin embargo, en el presente caso, a mayo ya se habían hecho cinco cortes, debido a que este fue un año bueno desde el punto de vista climático para la actividad de la henificación, donde el verano entró temprano en noviembre y el invierno entró tarde en mayo. Sin embargo, se produjeron 6560 pacas, casi las 7000 que de 5 ha en producción total, podrían lograrse según la tecnología bajo validación, aunque en finca, se lograron con menores tasas productivas, pero con más cortes. 
Cuadro 6. Comparación de costos y rentabilidad real del productor Floriberto Esquivel y la E.E.E.J.N., durante el primer ciclo de producción. Guanacaste, Costa Rica. 2000.

\begin{tabular}{|c|c|c|}
\hline RUBRO & COSTO FINCA ( $(\varphi)$ & COSTO E.E E.J.N. ( $(\varphi)$ \\
\hline Fertilizantes * & 516200,00 & 316634,00 \\
\hline Servicio de agua & 112500,00 & 112500,00 \\
\hline Manejo de riegos & 22500,00 & 22500,00 \\
\hline Servicio de henificación & 1312000,00 & 1400000,00 \\
\hline Almacenaje de pacas & 30000,00 & 32000,00 \\
\hline Control de malezas & 90000,00 & 90000,00 \\
\hline Establecimiento 10 años vida útil & 60250,00 & 77700,00 \\
\hline TOTAL & $2158.000,00$ & 2052361,50 \\
\hline Ingreso total neto & 1778000,00 & 2147638,50 \\
\hline Ingreso neto por hectárea & 355600,00 ** & 428500,00 \\
\hline
\end{tabular}

*Productor usó 5,2 qq y Estación experimental 4,0 qq de nitrato de amonio/ha/corte

${ }^{\star \star} A$ valores actualizados al 2011 el ingreso neto en finca sería de $\not 2296$ 000,00 - $\not 592$ 220,00 = $\$ 1703780,00$ (\$3 308).

Teniendo en cuenta las consideraciones apuntadas anteriormente y tomando los resultados de los cálculos comparativos como una buena aproximación, podemos decir, que los resultados a nivel de investigación en términos biológicos se pueden alcanzar en un $94 \%$ (6 560/ 7000 pacas) y en términos económicos en un $83 \%$ (\$355 600,00/ $ф 428500,00)$.

Se puede concluir, por lo tanto, que los resultados obtenidos en la estación experimental son reproducibles, en más de un $80 \%$, a nivel de finca comercial, con lo cual esta tecnología se debe transferir a los pequeños productores del Distrito de Riego Arenal Tempisque.

Esta conclusión está reforzada por las consideraciones hechas en la introducción del presente documento, en donde se describe el tipo de mercado de heno para crisis por clima, existente en el país, y como el heno de riego podría abrir la posibilidad de un mercado de calidad para la producción animal y capturar el mercado actual existente de pacas de mala calidad. El mercado podría ampliarse a clientes nuevos en fincas lecheras y ganado de engorde estabulado. Pero antes, para ello debe ponerse esta clase de producto en el mercado en cantidades apreciables para cambiar la cultura hacia un mercado por calidad.

Validación de la producción y calidad, del heno de pasto Transvala, como respuesta a la edad de rebrote y a la fertilización nitrogenada, bajo condiciones de riego.

\section{Contenido de materia seca del forraje.}

La fuente de nitrógeno, sea esta nitrato de amonio o urea y la aplicación en dosis iguales o incrementales, no afectaron el contenido materia seca del forraje, mientras que el nivel de fertilización de cualquiera de las fuentes de nitrógeno sí la afecto $(\mathrm{P}<0,001)$ como se observa en el Cuadro 7. 
Cuadro 7. Contenido materia seca del forraje según niveles de fertilización. Guanacaste, Costa Rica.2000.

\begin{tabular}{cccccccc}
\hline \multicolumn{7}{c}{ NIVELES DE FERTILIZACIÓN NITROGENADA (kg/ ha) } \\
\hline Variables & EEM $^{*}$ & $\mathbf{0}$ & $\mathbf{1 0 0}$ & $\mathbf{2 0 0}$ & $\mathbf{3 0 0}$ & $\mathbf{4 0 0}$ & $\mathbf{5 0 0}$ \\
\hline$\% \mathrm{MS}$ & 2,91 & $35,4_{\mathrm{a}}$ & $27,7_{\mathrm{b}}$ & $22,8_{\mathrm{c}}$ & $21,6_{\mathrm{c}}$ & $21,2_{\mathrm{c}}$ & $21,0_{\mathrm{c}}$ \\
\hline
\end{tabular}

* EEM = error estándar de la media

Medias con letras diferentes son diferentes entre sí $(\mathrm{P}<0,001)$.

Este resultado concordó con las observaciones hechas en anteriores estudios a nivel de finca experimental (Morales et al. 2006 a y b). En el análisis de estos resultados por regresión múltiple se mostró un efecto cuadrático ( $R 2=74 \%$ ) según la función $y=34,925-0,07956073 x+$ 0,00010798x2, (Figura 3).

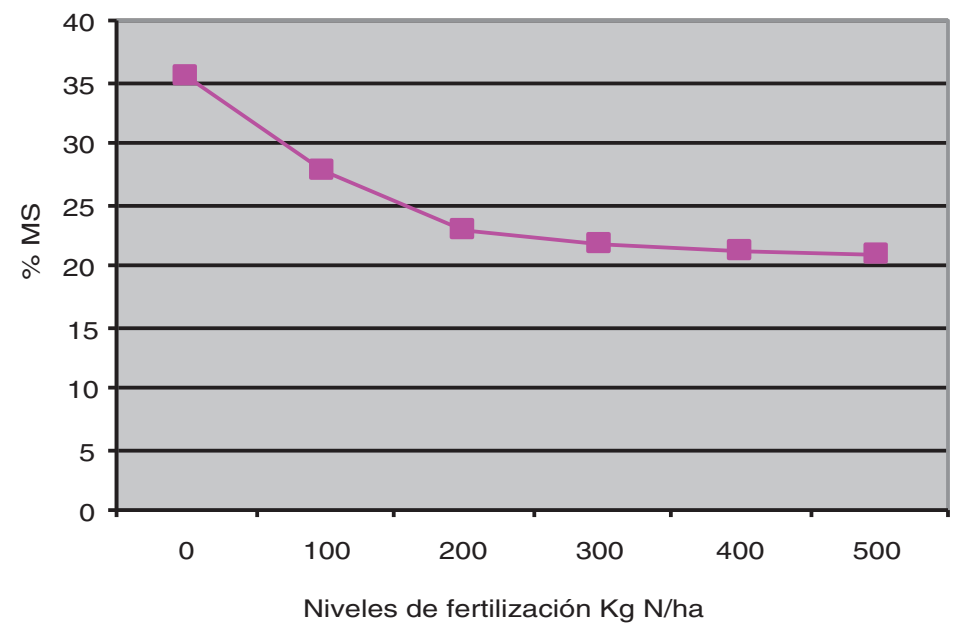

Figura 3. Respuesta del pasto Transvala a la fertilización nitrogenada. Guanacaste, Costa Rica. 2000.

A mayor nivel de fertilización, el contenido de materia seca del forraje disminuyó, entre 0 y $200 \mathrm{~kg}$ de $\mathrm{N} /$ ha la tasa de reducción es particularmente alta.

\section{Producción de forraje.}

Igual que en el punto anterior, la fuente y la dosificación del nitrógeno no afectaron la producción de forraje. El nivel de fertilización, por el contrario tuvo un fuerte efecto sobre este parámetro, (Cuadro 8).

Cuadro 8. Producción de forraje en base seca según niveles de fertilización. Guanacaste, Costa Rica. 2000.

NIVELES DE FERTILIZACIÓN NITROGENADA (kg/ ha)

\begin{tabular}{ccccccccc}
\hline Variables & EEM $^{*}$ & $\mathbf{0}$ & $\mathbf{1 0 0}$ & $\mathbf{2 0 0}$ & $\mathbf{3 0 0}$ & $\mathbf{4 0 0}$ & $\mathbf{5 0 0}$ & \\
\hline $\mathrm{kg} \mathrm{MS} / \mathrm{ha}$ & 990,80 & $3560,40 \mathrm{~d}$ & $4155,60 \mathrm{~d}$ & $5511,10 \mathrm{c}$ & $6643,10 \mathrm{~b}$ & $6873,60 \mathrm{ab}$ & 7634,90 & $\mathrm{a}$ \\
\hline
\end{tabular}

${ }^{\star} \mathrm{EEM}=$ error estandar de la media

Medias con letras diferentes son diferentes entre sí $(P<0,001)$. 
El aumento no fue significativo sino hasta el nivel de los $200 \mathrm{~kg}$ de $\mathrm{N} / \mathrm{ha}$, mientras que en la estación experimental se observaron este tipo de diferencias desde el nivel de los $100 \mathrm{~kg}$ de N/ ha, como se puede observar más fácilmente en el gráfico siguiente, Figura 4.

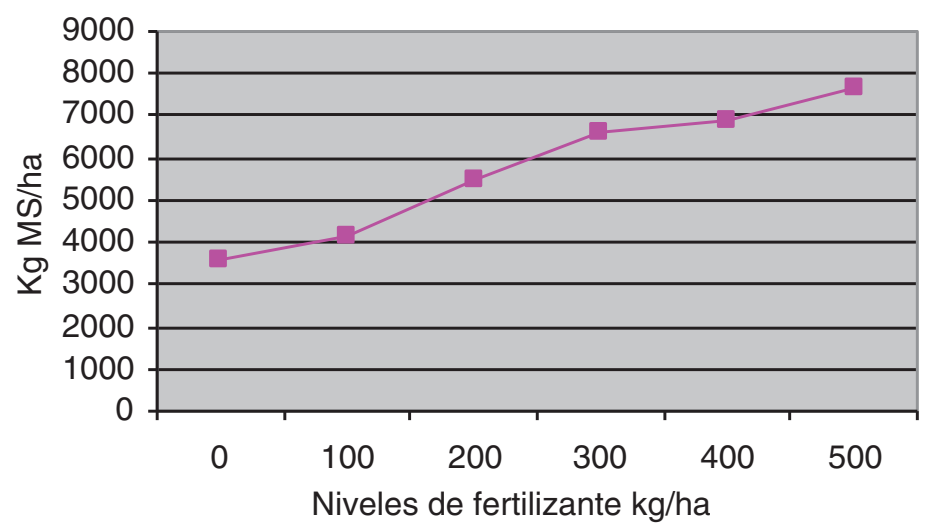

Figura 4. Respuesta del pasto Transvala a la fertilización nitrogenada. Guanacaste, Costa Rica. 2000.

En este estudio de validación en finca, aún a $500 \mathrm{~kg}$ de N/ ha no se lograron observar rendimientos decrecientes del fertilizante, que sí se pudieron notar bajo las condiciones de la estación experimental. Al contrario, se detectó una tendencia casi lineal en el comportamiento de este efecto $\left(R^{2}=54,80 \%\right)$, explicado por la función $y=3617,86+8,47 x$.

\section{Contenido de proteína cruda del forraje.}

La fuente y la dosificación del nitrógeno no afectaron el contenido de proteína del forraje. Como todas las observaciones de investigación anteriores (Morales et al. 2006 a y b), la proteína sí varió según el nivel de fertilización. Nada más que en este caso, el máximo contenido se alcanzó a los $200 \mathrm{~kg} \mathrm{~N} /$ ha, nivelándose de aquí en adelante (Cuadro 9).

Cuadro 9. Contenido de proteína cruda del forraje según niveles de fertilización. Guanacaste, Costa Rica. 2000.

NIVELES DE FERTILIZACION NITROGENADA (kg/ ha)

\begin{tabular}{cccccccc}
\hline Variables & EEM $^{*}$ & $\mathbf{0}$ & $\mathbf{1 0 0}$ & $\mathbf{2 0 0}$ & $\mathbf{4 0 0}$ & $\mathbf{5 0 0}$ & $\mathbf{3 0 0}$ \\
\hline$\% \mathrm{PC}$ & 0,91 & $6,08 \mathrm{c}$ & $7.42 \mathrm{~b}$ & $8,41 \mathrm{a}$ & $8,55 \mathrm{a}$ & $8,64 \mathrm{a}$ & $8,69 \mathrm{a}$ \\
\hline
\end{tabular}

${ }^{*} E E M=$ error estandar de la media

Medias con letras diferentes son diferentes entre sí $(P<0,001)$. 
Lo cual se puede observar claramente en el gráfico siguiente (Figurea 5).

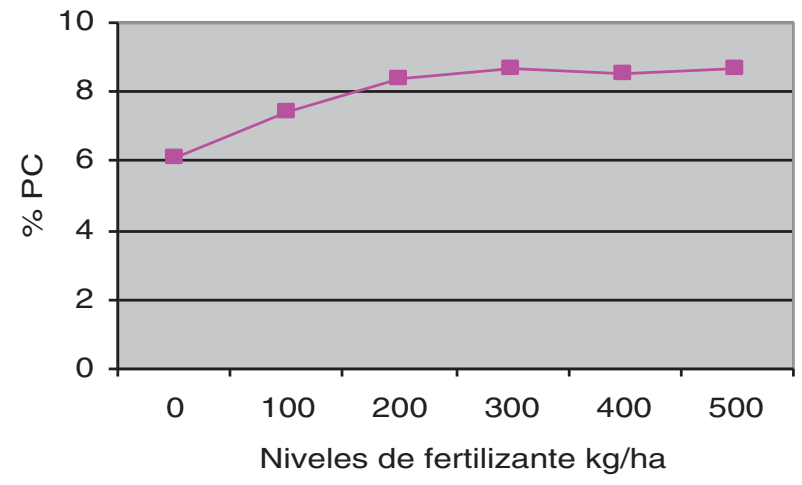

Figura 5. Respuesta del pasto Transvala a la fertilización nitrogenada. Guanacaste, Costa Rica. 2000.

\section{Contenido de fibra del forraje.}

El contenido de fibra neutro detergente no fue afectada por ninguna de las variables evaluadas, manteniéndose una media de $65,70 \%$ en todas las muestras.

Por el contrario, la fibra ácido detergente sí se vio afectada por el nivel de fertilización $(P<0,001)$. El comportamiento aparentemente lineal y positivo, sin embargo, no fue corroborado estadísticamente $\left(R^{2}=32.5 \%\right)$.
Análisis de los niveles de nitritos y nitratos en las aguas de percolación,

Toda actividad productiva tiene que ser socioeconómicamente viable, pero ante todo tiene que ser ambientalmente sostenible. Cuando se importan e incorporan insumos a la finca para mejorar la producción, se debe tener la certeza, de cuál debe ser el límite máximo de uso de ese insumo, para así mantenerse dentro de los límites inocuos para el ambiente y como consecuencia, para el ser humano y los animales. En este sentido, se debe ser aún más suspicaz cuando el insumo de que se trate, es de origen inorgánico como los agroquímicos, aunque las excretas de los animales y otras sustancias orgánicas podrían también aportar a la concentración de sustancias tóxicas en el medio.

Los fertilizantes inorgánicos y en particular los nitrogenados vinieron a revolucionar la agricultura moderna (la revolución verde). Como tales son de importancia capital, sin embargo, se reconocen los efectos contaminantes de su abuso. Los nitritos $\left(\mathrm{NO}_{2}\right)$ y los nitratos $\left(\mathrm{NO}_{3}\right)$ son los dos derivados más importantes de la aplicación de fertilizantes.

La aplicación de fertilizantes nitrogenados a pastos, toma varias direcciones, absorción

Cuadro 10. Contenido de fibra ácido detergente del forraje según niveles de fertilización. Guanacaste, Costa Rica. 2000.

NIVELES DE FERTILIZACIÓN NITROGENADA (kg/ha)

\begin{tabular}{cccccccc}
\hline Variables & EE & $\mathbf{0}$ & $\mathbf{1 0 0}$ & $\mathbf{2 0 0}$ & $\mathbf{3 0 0}$ & $\mathbf{4 0 0}$ & $\mathbf{5 0 0}$ \\
\hline$\%$ FAD & 1,94 & $46,09 \mathrm{~d}$ & $48,32 \mathrm{c}$ & $50,97 \mathrm{~b}$ & $51,63 \mathrm{ab}$ & $52,67 \mathrm{ab}$ & $52,97 \mathrm{a}$ \\
\hline
\end{tabular}

Medias con letras diferentes son diferentes entre si $(\mathrm{P}<0,001)$. EE = error estándar. 
por las raíces de las plantas, inmovilizado en la materia orgánica del suelo o disperso al ambiente. Cuatro diferentes procesos juegan un rol en la pérdida de nitrógeno de la zona radicular: desnitrificación, resultando en emisiones de $\mathrm{N}_{2}$ y $\mathrm{N}_{2} \mathrm{O}$ a la atmósfera, volatilización de $\mathrm{NH}_{3}$, lixiviado como $\mathrm{NO}_{3}$ en el subsuelo ó perdido por escorrentía.

Los nitratos y nitritos son solubles en agua y por lo tanto se mueven con el agua. Los nitratos, menos tóxicos que los nitritos, se presentan en el agua o alimentos en concentraciones mucho más altas. El nitrato agregado al ó producido en el suelo, puede lixiviarse o lavarse por escorrentía. Los excesos de nitratos se les encuentran acumulados en las aguas del subsuelo en áreas bajas. La agencia de protección ambiental de los Estados Unidos tiene dentro de los estándares de agua potable un máximo de $10 \mathrm{mg} / \mathrm{L}$ de nitratos y $1 \mathrm{mg} / \mathrm{L}$ de nitritos (Undersander 2001), como concentraciones seguras, tanto para humanos como para animales. Dentro de las fuentes de contaminación de aguas con este componente está la fertilización.

También pueden ocurrir altas concentraciones de nitratos en los forrajes a consecuencia de la aplicación de fertilizantes, así como a la cantidad de fertilizante aplicado, y a otros factores como temperatura y tiempo transcurrido entre la aplicación y el muestreo. En algunas especies de pastos en otros países se ha observado poca acumulación de nitratos cuando la tasa de fertilización es menor a 300 $\mathrm{kg}$ de N/ ha/ año. Sobre tasas de $450 \mathrm{~kg}$ de $\mathrm{N} / \mathrm{ha} /$ año si se observan concentraciones críticas. Una concentración de cerca de 0,4\% (4 000 ppm) de N-NO3 es considerado potencialmente tóxico para rumiantes (Whitehead 1995). Los métodos para valorar las cantidades de $\mathrm{N}$ lixiviado del suelo pueden ser mediante lisímetros, el muestreo de suelo o solución del suelo a varias profundidades (método utilizado en el presente estudio) ó el análisis de las aguas de drenajes en el campo (Whitehead 1995).

En el presente estudio, las muestras para determinar la concentración de nitratos en la solución del suelo se tomaron a los 4,14 y 15 días después de la aplicación del nitrato de amonio (NH4NO3), durante tres aplicaciones continuas separadas 15 días entre sí (20 marzo, 4 abril y 20 abril). La idea fue la de tener la oportunidad de observar el comportamiento de los nitratos en esos rangos de tiempo. Aunque se encontraron efectos principales debido a fecha de muestreo y niveles de fertilización, estos quedaron relegados por la interacción entre fecha y nivel de fertilización $(P<0.01)$, como se puede observar en la Figura 6.

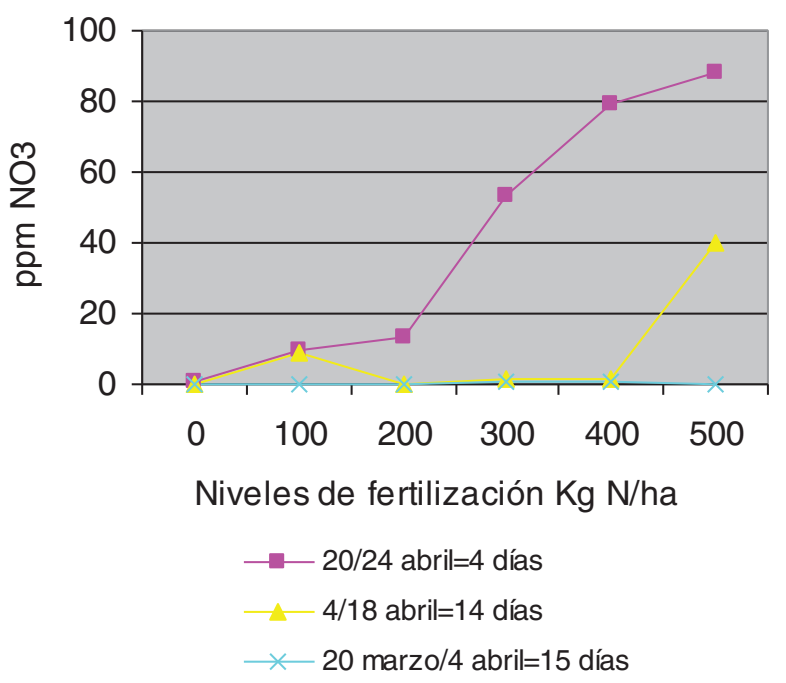

Figura 6. Interacción entre el intervalo de muestreo de los niveles de fertilización y el contenido de los nitratos del suelo. Guanacaste, Costa Rica. 2000.

Cuatro días después de la aplicación del fertilizante, el efecto, particularmente de los niveles arriba de los $200 \mathrm{~kg} \mathrm{~N} / \mathrm{ha}$, alcanzaron concentraciones muy altas de nitritos en la solución del suelo. Sin embargo, 14 días después de la aplicación del fertilizante, las concentraciones de nitratos en la solución del suelo vuelven a ser bajas, con excepción del nivel de $500 \mathrm{~kg}$ de N/ ha, que permaneció arriba de los rangos mínimos aceptados. Esta última observación no fue consistente, sin embargo, pues no se repitió en el otro muestreo de 15 días. 
El resultado observado a los cuatro días del muestreo, indica un comportamiento casi lineal (R2 $=62,60 \%$ ) de los niveles de fertilización y la concentración de nitratos en la solución del suelo ( Figura 7).

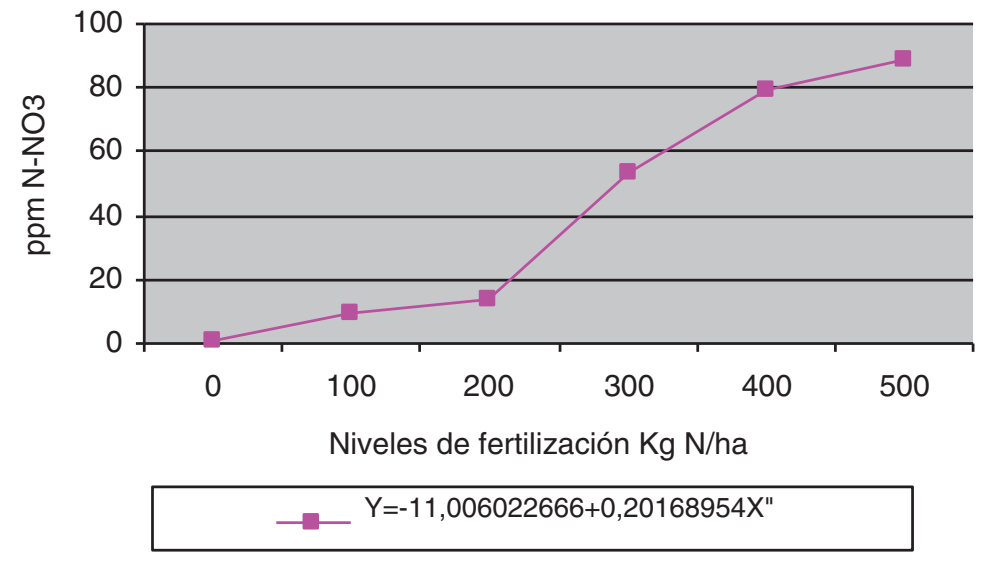

Figura 7. Concentración de nitratos en la solución del suelo cuatro días después de la aplicación de nitrógeno. Guanacaste, Costa Rica. 2000.

También se debe considerar la posibilidad de que, en el muestreo a los cuatro días de aplicación del fertilizante, hubiera un efecto acumulativo ya que los muestreos se realizaron en el siguiente orden:

Cuadro 11.Características de los muestreos de solución del suelo. Guanacaste, Costa Rica. 2000.

\begin{tabular}{cccc}
\hline $\begin{array}{c}\text { Fecha } \\
\text { Aplicación } \mathbf{N}\end{array}$ & $\begin{array}{c}\text { Fecha de } \\
\text { Muestreo }\end{array}$ & $\begin{array}{c}\text { Aplicaciones } \\
\text { Acumuladas }\end{array}$ & $\begin{array}{c}\text { Nivel Acumulado N } \\
\text { (dosis crecientes) }\end{array}$ \\
\hline 20 marzo & 4 abril = 15 días & 1 & $0,17,33,50,67,83$ \\
4 abril & 18 abril = 14 días & 2 & $0,50,100,150,200,250$ \\
20 abril & 24 abril $=4$ días & 3 & $0,100,200,300,400,500$ \\
\hline
\end{tabular}

Más trabajos en esta materia se requieren, para dar un seguimiento de mediano plazo, al menos, y así ponerle los límites adecuados, para que esta sea una tecnología ambientalmente sostenible. Como medidas de precaución para la protección ambiental y según indican los resultados hasta aquí obtenidos, la tecnología del heno de pasto Transvala bajo riego y fertilizado, no debiera trabajar con niveles de fertilización nitrogenada arriba de los $100 \mathrm{~kg}$ de N/ ha/ corte.

\section{CONCLUSIONESY RECOMENDACIONES}

El presente trabajo de validación de la tecnología, para la producción de heno de pasto Transvala de calidad superior, en sistemas bajo riego, abordó la evaluación a nivel de finca desde el método de siembra del cultivo y sus costos, el establecimiento hasta producción, y los procesos de manejo de lotes para henificación, costos y rentabilidad.
Se modificaron prácticas de preparación del suelo, acordes con la infraestructura existente en el Distrito de Riego.

También se validó la respuesta del cultivo a la fertilización nitrogenada, al manejo de 45 a 50 días de rebrote, en términos de producción de forraje y valor nutritivo. Algunas variantes de la tecnología original también se incorporaron como fue la comparación entre dos fuentes de nitrógeno, urea y nitrato 
de amonio, dosificación del fertilizante en tres aplicaciones iguales y en cantidades incrementales. También se adaptó el número de riegos a las condiciones físicas de los suelos. Se corroboraron los resultados de la Estación Experimental plenamente en lo que se refiere a lo mencionado en este párrafo.

Finalmente, se evaluó el efecto de las prácticas recomendadas por la tecnología en términos de su efecto en la concentración de sustancias contaminantes en la solución del suelo, particularmente en forma de nitratos. El trabajo tomo dos años en su ejecución 20002001.

Los resultados de este proceso de evaluación indicaron que la tecnología desarrollada en la estación Enrique Jiménez Núñez, para la Industrialización del heno de pasto Transvala en el Distrito de Riego, se aplicó comercialmente con ligeras modificaciones.

El método de establecimiento se mantuvo igual, más bien reduciendo un poco la intensidad de ciertas labores, a causa de la oportunidad de este cultivo para utilizar la infraestructura del arroz sin mayores problemas, lo cual causa una favorable reducción en costos de establecimiento. Se reproduce la observación de la estación experimental, de que la tecnología tiene naturalmente, un autocontrol de malezas debido al corte frecuente del cultivo, el cual las malezas comunes de la zona no soportan. Desde luego, deben existir unos controles mínimos, particularmente en invierno, cuando el cultivo está prácticamente inactivo.

Se logró comprobar los rendimientos, calidad del producto, costos y rentabilidad de la actividad sobre el $80 \%$, aún en conocimiento de que esta primera etapa en que se evaluó el sistema en finca comercial está en fase de consolidación y desarrollo.

Finalmente, el proceso de validación permitió una evaluación que no se había hecho antes y que consistió en determinar que los niveles de fertilizante a aplicar, no deben sobrepasar los $200 \mathrm{~kg}$ de N/ ha/ corte. Esto ya que se evidenció la posibilidad de incrementar la concentración de sustancias contaminantes como los nitratos, a consecuencia de la fertilización sobre esas cantidades y que como precaución para la protección ambiental es recomendable quedarse, con los niveles de respuesta biológica y económica indicados por los estudios previos (Morales et al. 2006 a y b), al presente, que recomendaron tasas de fertilización nitrogenada entre 65 y $100 \mathrm{~kg}$ de $\mathrm{N} / \mathrm{ha} /$ corte.

En conclusión el estudio mostró la factibilidad biológica, económica y ambiental para proceder a transferir esta tecnología. Es precisamente las necesidades de nuevas opciones tecnológicas de los pequeños productores del DRAT y el momento oportuno en que ésta se presenta, que la da la viabilidad social a la misma.

\section{LITERATURA CITADA}

EPA (U.S. Environmental Protection Agency). 2003. Ruminant Livestock and the Global Environment. Disponible en: http:/www. epa.gov/rlep/sustain.htm. 3 p.

Goering, H. K., van Soest, P. J. 1970. Forage fibre analysis. Agricultural handboock № 379 . USDA, Washington, D.C.

Gomez, V. O. 2002. Estudios semidetallados de suelos del DRAT, Guanacaste. San José, C.R. Instituto Nacional de Innovación y Transferencia en Tecnología Agropecuaria. P 30.

Hancock, J.R., Hargreaves, H.G. 1977. Precipitación, clima y potencial para producción agrícola en Costa Rica. International Irrigation Center. Agricultural and Irrigation Engineering Dept. Utah State University. P 160.

Morales, J., Acuña, V. y Cruz, A. 2003. Industrialización del Heno de Calidad en Sistemas Bajo Riego en Costa Rica. INTA. Costa Rica. P 79.

Morales, J, Cruz, A., Acuña, V, 2006.a Efecto del estado de madurez y la fertilización nitrogenada sobre la producción y valor nutritivo del pasto transvala (Digitaria decumbens cv. Transvala) para henificación bajo condiciones de secano. Alcances Tecnológicos. Año IV, № 1. p 37-44 
Morales et al::Validación de la Respuesta del Pasto Transvala (Digitaria Eriantha) en Producción y Calidad de Heno Bajo Riego

Morales, J, Acuña, V, Cruz, A., 2006.b Efecto de la fertilización nitrogenada sobre la producción y valor nutritivo del pasto transvala (Digitaria decumbens cv. Transvala) para henificación, bajo condiciones de riego. Alcances Tecnológicos. Año IV, № 1. p 45-51.

Morales, J, Acuña, V, Cruz, A. 2003. Industrialización del heno de calidad en sistemas bajo riego en Costa Rica. Imprenta Nacional. Ministerio de Agricultura y Ganadería). p 77.

Tisdale, S.L., Nelson, W.L., Beaton, J.D.. 1985. Soil fertility and fertilizers. 4 ed. Nueva York, USA. Macmillan. P 560.
Undersander, D. 2001. Does forage quality pay. Proceedings of the AMER. Forage and sGrasland. Council. Springdale, AR. Texas, USA. P120-125

Van Soest, P.J. 1967. Development of a comprehensive system of feed analyses and its application to forages. J. Anim. Sci. 26:119.

Whitehead, D.D. 1995. Grassland Nitrogen. CAB Intl. England. p397.

ANEXO.

CUADRO 1. Costos de siembra y establecimiento de pasto Transvala (Digitaria eriantha) en el Distrito de Riego Arenal-Tempisque. Bagaces, Guanacaste. 2011.

\begin{tabular}{|c|c|}
\hline Componentes & Precios Ajustados \\
\hline $\begin{array}{l}\text { 1. Servicio de mecanización } \\
\text { - } 1 \text { pase rastra } \\
\text { - } 2 \text { pases de fangueadora }\end{array}$ & $\Varangle 100000,0$ \\
\hline $\begin{array}{l}\text { 2. Semilla } \\
\text { - Semilla } \\
\text { - Corte (4 peones X } 8.7 \mathrm{hr} \times \varnothing 1200) \\
\text { - Transporte semilla }\end{array}$ & $\begin{array}{l}\text { c } 176700,0 \\
\cdot 100000,0^{\star} \\
\cdot 43200,0 \\
\cdot 33500,0\end{array}$ \\
\hline $\begin{array}{l}\text { 3. Siembra } \\
\text { - Mano de obra ( } 7 \text { peones x } 20 \mathrm{hr} \times \notin 1066) \\
\text { - Transporte peones }\end{array}$ & $\begin{array}{l}\text { c } 181500,0 \\
\cdot 168000,0 \\
\cdot 13500,0\end{array}$ \\
\hline $\begin{array}{l}\text { 4. Fertilización } \\
\text { - Fertilizante (2.5 sacos 10-30-10 X \& 24.000) } \\
\text { - Aplicación }\end{array}$ & $\begin{array}{l}\text { \& } 60600,0 \\
\cdot 47500,0 \\
-3100,0\end{array}$ \\
\hline $\begin{array}{l}\text { 5. Control malezas } \\
\text { - Hierbicidas (2,4-D+Ally+Pega+Round-up) } \\
\text { - Aplicación }\end{array}$ & $\begin{array}{l}\text { C } 54620,0 \\
\cdot 20820,0 \\
\cdot 33800,0\end{array}$ \\
\hline 6. Limpia de bordos (chapia) & c 18800,0 \\
\hline COSTO TOTAL / ha & c 592220,0 \\
\hline
\end{tabular}

No hay costos por hechura de bordos, rastra o rufa porque el establecimiento de este cultivo para heno utiliza la misma infraestructura existente para arroz y las siembras se recomiendan ahora hacerlas durante el invierno, porque hay mayor disponibilidad de semilla y el establecimiento es mucho más efectivo y seguro en este periodo pues la semilla es de mayor calidad y pega mejor. Precios : * $₫ 2000 /$ paca semilla, hora jornal $₫ 1200$, saco 10-30-01 $₫ 19000,0$ mesulfuron methil $15 \mathrm{gr} / \mathrm{ha}$ o Ally $\varnothing 1800$, pega ф3 000,0, 2,4-D $\phi 4.680 /$ lt, Round-up $ф 3.330 /$ It. 
Cuadro 2. Costos operativos de producción de heno de pasto Transvala (Digitaria eriantha) solo bajo riego en el Distrito de Riego Arenal-Tempisque. Bagaces, Guanacaste. 2011.

\begin{tabular}{|c|c|c|}
\hline Componentes & Costos corte diciembre & Costos cortes riego \\
\hline $\begin{array}{l}\text { 1. Fertilización } \\
\text { •Transvala }\end{array}$ & 0,0 & c 81400,0 \\
\hline $\begin{array}{l}\text { 2. Canon SENARA por agua } \\
\text { • } \not 20.000,0 / \text { ha /semestre }\end{array}$ & c 20000,0 & c 20000,0 \\
\hline $\begin{array}{l}\text { 3. Mantenimiento de bordos } \\
\text { - Mano de obra ( } 3 \text { días peón) }\end{array}$ & c 21000,0 & C 21000,0 \\
\hline $\begin{array}{l}\text { 4. Manejo de riegos } \\
\text { - Mano de obra }\end{array}$ & $\varnothing 0,0$ & c 33800,0 \\
\hline $\begin{array}{l}\text { 5. Servicio de henificación } \\
\qquad \not \subset 550.0 \text { / paca }\end{array}$ & $\begin{array}{l}400 \times 550= \\
\varnothing 220000,0\end{array}$ & $\begin{array}{l}600 \times 550= \\
\varnothing 330000,0\end{array}$ \\
\hline 6. Almacenaje de pacas $₫ 150 /$ paca & \& 60000,0 & $\varnothing 90000,0$ \\
\hline 7. Control de malezas & c 21000,0 & ф 7000,0 \\
\hline 8. Inversión inicial (10\%) & ф 29610,0 & ф 29610,0 \\
\hline COSTO TOTAL / ha/subsistema & c 371610,0 & c 612810,0 \\
\hline COSTO TOTAL/ha ciclo producción & \multicolumn{2}{|c|}{ c 984.420,0 } \\
\hline Costo /paca & c 929,0 & c 1021.4 \\
\hline $\begin{array}{l}\text { Precio mínimo de mercado / } \\
\left(30 \% \text { utilidad)/paca }{ }^{1}\right.\end{array}$ & c 1327,0 & c 1459,0 \\
\hline $\begin{array}{l}\text { Precio promedio ponderado } \\
\text { para periodo ( } 30 \% \text { utilidad mínima) }\end{array}$ & \multicolumn{2}{|c|}{ c 1406,2 } \\
\hline
\end{tabular}

${ }^{1}$ Costos sobre la base de 4 cortes (400 pacas secano en diciembre y 3 cortes - 200/corte - de verano con riego), 1000 pacas producidas en total durante el ciclo por hectárea, servicio de maquinaria por paca $₫ 550,0$, fertilización por corte 4 sacos nitrato de amonio y bianual de 2 sacos de 10-30-10 , \$21000 aplicación del fertilizante. Cálculos con base a producción mínima aceptable, la máxima alcanzable es de 1400 en transvala solo.Bajo estas condiciones de máxima producción el precio ponderado para una utilidad mínima del $30 \%$ es de $\varnothing 1290,2$, es decir el negocio aguantaría una reducción de precios de hasta un $10 \%$, comparado con la producción de 1000 pacas/ha y aún así la rentabilidad por ha aumentaría en un $28,5 \%$ pasando de $\varnothing 421780$ a $\varnothing 542.000,0$ por hectárea. Hasta 2009 y particularmente el 2008 se pagaron precios en finca de hasta $\varnothing$ 2000/paca, es decir el negocio fue excelente, sin embargo, en el 2010 y a consecuencia de esos precios, muchos ganaderos no productores de heno, cosecharon heno, además de una finca grande que había entrado en el negocio en el 2007, crearon una sobreproducción trayéndose el precio de $\phi 1000$. 